\title{
Check-list de Plumbaginaceae e Polygonaceae do estado de Mato Grosso do Sul, Brasil
}

\author{
Raquel Carvalho', Bruna Zancanelli Fetter', Efigênia de Melo² \& Maria Ana Farinaccio³
}

\author{
' Universidade Federal de Mato Grosso do Sul, Instituto de Biociências, Cidade Universitária, s/nº, Caixa Postal 549 , \\ CEP 79070-900. Campo Grande, MS, Brasil. raquelcrvlh@hotmail.com \\ 2 Universidade Estadual de Feira de Santana, Departamento de Ciências Biológicas, Câmpus Universitário, Caixa Postal 294 \\ CEP 44036-900. Feira de Santana, BA, Brasil \\ ${ }^{3}$ Universidade Federal de Mato Grosso do Sul, Câmpus Pantanal, avenida Rio Branco, n 1270, CEP 79304-902. Corumbá, MS, Brasil.
}

Recebido em 27.IX.2014

Aceito em 22.VIII.2016

DOI 10.21826/2446-8231201873s308

RESUMO - Plumbaginaceae e Polygonaceae serão aqui tratadas por nomearem um grupo irmão e pela pouca representatividade da primeira. As duas famílias são compostas geralmente por ervas, com folhas simples e alternas e fruto do tipo aquênio. A elaboração deste checklist partiu da publicação de Dubs, foi complementado com levantamento no acervo do Herbário da Universidade Federal de Mato Grosso do Sul (CGMS), no banco de dados do projeto SpeciesLink e na lista de Espécies da Flora do Brasil. Os resultados apontam que não houve alteração no número de táxons de Plumbaginaceae para o Brasil nem para o estado, ou seja, ainda há a ocorrência de apenas um gênero e uma espécie subordinada à ele. O número de Polygonaceae também não se alterou para o Brasil, o qual permaneceu 93 espécies distribuídas em nove gêneros, no entanto, para o Mato Grosso do Sul foram citadas 24 espécies e, após esse estudo, 27.

Palavras Chaves: Caryophyllales, Chaco, Pantanal

ABSTRACT - Checklist of Plumbaginaceae and Polygonaceae in Mato Grosso do Sul state, Brazil. Plumbaginaceae and Polygonaceae will be discussed in this study because they are sister groups, and due to little representation of the first. Both families are usually composed of herbs, with simple and alternate leaves, and achene as the fruit type. This checklist is based on Dubs publication, supplemented with the University of Mato Grosso do Sul (CGMS) Herbarium collection, and the databases of SpeciesLink and Species of the Flora of Brazil list. Our results showed that there were no changes in the number of taxa in Plumbaginaceae for Brazil nor for the state, which means that only one genus with one subordinated species occurs . The number of Polygonaceae also did not change for Brazil (93 species in nine genera); however, for Mato Grosso do Sul, 24 species had previously been cited, and after this study, there are 27.

Keywords: Caryophyllales, Chaco, Pantanal

\section{INTRODUÇÃO}

A família Plumbaginaceae emerge como grupo irmão de Polygonaceae, na ordem Caryophyllales de acordo com APGIII (2009), Olmstead et al. (1992) e Moore et al. (2011), assim sendo, e também, por Plumbaginaceae ser pouco representada no Brasil, preferimos tratar as duas famílias neste estudo.

Os representantes de Plumbaginaceae são, geralmente, ervas perenes, com folhas simples e alternas sem estípulas (Farinaccio \& Nascimento 2005). Sua inflorescência é do tipo cimosa ou racemosa, com flores actinomorfas, diclamídeas, bissexuadas e de ovário súpero. Os frutos são do tipo aquênio ou cápsula basalmente circuncisa (Souza \& Lorenzi 2008).

Esta família possui cerca de 30 gêneros e 800 espécies distribuídas pelo mundo (Souza \& Lorenzi 2008), mas principalmente em ambientes secos e salinos, especialmente no mediterrâneo e Ásia central (Farinaccio \& Nascimento
2005). Plumbaginaceae não é endêmica no Brasil, e está representada por dois gêneros, Plumbago L. e Limonium Mill., com uma espécie cada uma, encontradas, geralmente, na Amazônia, Caatinga e Mata Atlântica (Zappi 2012).

Os indivíduos da família Polygonaceae são compostos normalmente por ervas, arbustos ou árvores, com folhas simples, alternas e possuem estípulas (ócreas) caducas ou persistentes. A inflorescência é comumente em tirsos racemosos, paniculados ou fasciculados, com brácteas persistentes denominadas ocréolas (Melo 2000). As flores são actinomorfas, monoclamídeas ou diclamídeas, unissexuadas ou bissexuadas e de ovário súpero (Souza \& Lorenzi 2008). O fruto é simples, indeiscente e normalmente do tipo aquênio envolto pelo cálice persistente seco ou carnoso (Melo 2000).

Esta família apresenta 800 (Oliveira et al. 2008) a 1100 espécies (Souza \& Lorenzi 2008) em cerca de 40 gêneros, distribuídas nas regiões tropicais temperadas e subtropicais (Oliveira et al. 2008). De acordo com Melo 
(2012), no Brasil são encontrados e aceitos nove gêneros e 93 espécies distribuídas nos domínios fitogeográficos: Amazônia, Caatinga, Cerrado, Mata Atlântica e Pantanal.

Principais lacunas de conhecimento

Devido ao baixo número de espécie de Plumbaginaceae no Brasil, não são conhecidos grupos de pesquisa que trata especificamente sobre essa família. Por outro lado, a Professora Doutora Efigênia de Melo se destaca no estudo de Polygonaceae no Brasil, pela Universidade Estadual de Feira de Santana (UEFS).

Para o melhor conhecimento das famílias, em especial Polygonaceae, será necessário um esforço maior de coleta no estado, principalmente nos locais menos amostrados. As espécies de Triplaris e Ruprechtia são muito mal conhecidas no Brasil todo. Além disso, trabalhos na área de Ecologia de populações e talvez uma revisão taxonômica para o Brasil, pois o trabalho de Pendry (2003) não é suficiente, no que se refere às espécies brasileiras.

Principais acervos e Perspectivas de pesquisa para os próximos 10 anos

Foi verificado um baixo número de coletas de Plumbaginaceae. Para Polygonaceae, os principais herbários onde foram encontrados vouchers desta família coletados no Mato Grosso do Sul são, primeiramente, CGMS, seguido de CPAP (Embrapa Corumbá), HUEFS e UB.

O levantamento de Ruprechtia e Triplaris para o Mato Grosso do Sul, seria uma interessante dissertação de mestrado. Na área de ecologia, uma abordagem sobre ecologia de populações, associado à taxonomia dos grupos seria uma importante contribuição. Além disso, a interação entre Triplaris e formigas também poderia ser explorada nesta área.

\section{MATERIAL E MÉTODOS}

A elaboração desta lista partiu da publicação de Dubs (1998) que representa uma síntese para as Angiospermas do estado do Mato Grosso, quando este ainda incluía em seus limites o estado de Mato Grosso do Sul. Os dados foram complementados com um levantamento no acervo do Herbário da Universidade Federal de Mato Grosso do Sul (CGMS), sendo este o herbário base para a citação dos espécimes testemunhos (vouchers) e o maior acervo do estado para os grupos aqui tratados. Também foi consultado os bancos de dados do projeto SpLink (2012). A partir daí foi feita uma comparação com a Lista de Espécies da Flora do Brasil - LEFB (Forzza et al. 2012).

A abreviação dos nomes dos autores de táxons e citação das obras originais de publicações dos nomes científicos seguiu a proposta no site Tropicos (2012), do Missouri Botanical Garden, mantendo, assim, um mesmo padrão. Para a elaboração da lista dos táxons, foram adotados os conceitos mais recentes para os grupos aqui tratados (e.g. Moore et al. 2011). Os acrônimos dos herbários estão em acordo com Thiers (2008).

\section{RESULTADOS E DISCUSSÃO}

Os resultados obtidos representam uma atualização do conhecimento sobre as Plumbaginaceae e as Polygonaceae do Mato Grosso do Sul. Durante a elaboração deste checklist, foram detectados novos registros para o estado. O número de táxons de Plumbaginaceae e Polygonaceae, não sofreu alteração para o Brasil, e para o Mato Grosso do Sul, Polygonaceae passou de 24 para 27 espécies.

Plumbaginaceae

Plumbago L. in Sp. Pl. 1: 151. 1753.

As espécies desse gênero são normalmente arbustos e subarbustos perenes (Farinaccio \& Nascimento 2005), as quais têm sido usadas na medicina popular como antirreumático, purgativo, contra sífilis e dores de dente (Paiva et al. 2002). Os resultados obtidos concordam com a LEFB (Zappi 2012). No Mato Grosso do Sul, a única espécie que ocorre é $P$. scandens L, encontrada na Floresta Estacional Decidual no município de Nioaque.

Plumbago scandens L., Species Plantarum, Editio Secunda 1: 215-216. 1762.

Material examinado: L.C.S. Magalhães 31 (CGMS)

Polygonaceae

Antigonon Endl. in Gen. Pl. 4: 310. 1837.

Suas espécies são, geralmente, ervas perenes, distribuídas desde o México até a América Central e muito cultivadas como ornamentais na América do Sul (Kubitzki et al. 1993). De acordo com LEFB (Melo 2012), uma única espécie ocorre no Brasil, A. leptopus Hook. \& Arn.. A espécie é cultivada por todo o país e ocorre de forma subespontânea, principalmente na margem de estradas, próximo às construções.

Antigonon leptopus Hook. \& Arn. The Botany of Captain Beechey's Voyage 308-309, t. 69. 1841[1838]. (Dec 1838) Material examinado: P.R. Teodósio s.n. (CGMS 09987)

Coccoloba P. Browne in Civ. Nat. Hist. Jamaica 209-210. 1756.

Esse é o gênero mais representativo no Brasil (Melo 2000, 2003, Oliveira et al. 2008), distribuído desde a América Central até o Peru e a Bolívia (Cornejo \& Janovec 2010). A maioria de suas espécies ocorre em matas e apresenta ampla distribuição (Melo 2004), sendo relativamente comuns em florestas (Souza \& Lorenzi 2008). Muitas dessas espécies são úteis do ponto de vista etnomedicinal (Oliveira et al. 2008), comumente utilizadas no tratamento de febre, estresse, insônia, anemia, diarreia, problemas menstruais, gonorreia e hemorroida (Tsuboy et al. 2010, Correa \& Penna 1969 apud Barros et al. 2011). No Brasil há cerca de 40 espécies aceitas desse gênero, dentre essas, apenas 10 foram citadas para o Mato Grosso do Sul segundo a LEFB (Melo 2012), distribuídas no Chaco, Pantanal e Cerrado, a maioria em solo arenoso ou 
argiloso. Com esse estudo não foi confirmada a ocorrência de C. lucidula nem C. marginata. Por outro lado, $C$. paraguariensis, por equívoco, não foi citada na LEFB, mas, já havia sido referida para o Mato Grosso do Sul por Melo 2003.

Coccoloba argentinensis Speg. Physis. Revista de la Sociedad Argentina de Ciencias Naturales 3: 176. 1916. Material examinado: E. Melo 4228 (HUEFS)

Coccoloba cujabensis Wedd. Annales des Sciences Naturelles; Botanique, sér. 3 13: 259. 1849.

Material examinado: Damasceno-Junior, G.A. 3123 (CGMS, COR)

Coccoloba declinata (Vell.) Mart. Flora 20(Beibl. 2): 90. 1837. Coccoloba confusa R.A. Howard Journal of the Arnold Arboretum 41: 223. 1960.

Material examinado: Ratter J.A. 5107 (UB)

Coccoloba densifrons C. Mart. ex Meisn. Flora Brasiliensis 5(1): 26, pl. 7. 1855.

Material examinado: Hatschbach, G. 37432 (NYBG_BR)

Coccoloba guaranitica Hassl. Repertorium Specierum Novarum Regni Vegetabilis 14: 161. 1915.

Material examinado: D.R.C. Padilha 35 (CGMS)

Coccoloba mollis Casar. Novarum Stirpium Brasiliensium Decades 8: 72.1844.

Material examinado: W.M.Ramos 442 (CGMS)

Coccoloba obtusifolia Jacq. Enumeratio Systematica Plantarum, quas in insulis Caribaeis 19. 1760.

Material examinado: França, F. 4233 (NYBG_BR)

Coccoloba paraguariensis Lindau. Botanische Jahrbücher für Systematik, Pflanzengeschichte und Pflanzengeographie 13: 218, pl. 5. 1890. Coccoloba spinescens Morong, Annals of the New York Academy of Sciences 7: 212. 1893. Material examinado: E.P. Seleme 289 (CGMS)

Coccoloba parimensis Benth. London Journal of Botany 4: 626. 1845. Coccoloba ochreolata Wedd, Annales des Sciences Naturelles; Botanique, sér. 3, 13: 259. 1850.

Material examinado: R.S. de Arruda 21/126 (CGMS)

Polygonum L. in Sp. Pl. 1: 359-365. 1753.

Espécies de Polygonum são consideradas plantas invasoras (Jácome et al. 2004), e muitas delas são conhecidas como “erva-de-bicho" (Cardoso et al. 2006). Várias espécies são utilizadas em diferentes partes do mundo no tratamento de muitas doenças, incluindo infecções da pele, diarreia, hemorroida, insônia e doenças do coração, e também, na confecção de cosméticos (Alves et al. 2001, Cardoso et. al. 2006, Jácome et al. 2004). Polygonum contém cerca de 200 espécies, distribuídas principalmente nas regiões temperadas do hemisfério Norte (Kubitzki et al. 1993, Melo 1996). Nossos resultados concordam parcialmente com a LEFB (Melo 2012), havendo 16 espécies aceitas no Brasil, porém, foram encontradas somente oito no Mato Grosso do Sul, estando de fora a P. glabrum. Grande parte dos materiais testemunhos foi coletada no Pantanal e em outras áreas inundáveis.

Polygonum acuminatum Kunth. Nova Genera et Species Plantarum (quarto ed.) 2: 178. 1817[1818]. (Feb 1818)

Material examinado: M. Rocha 218 (CGMS)

Polygonum ferrugineum Wedd. Annales des Sciences Naturelles, Botanique, série 3, 13: 252. 1849.

Material examinado: T.H. Stefanello 159 (CGMS)

Polygonum hispidum Kunth. Nova Genera et Species Plantarum (quarto ed.) 2: 178. 1817[1818]. (Feb 1818)

Material examinado: M. Rocha 102 (CGMS)

Polygonum hydropiperoides Michx. Flora BorealiAmericana 1: 239. 1803.

Material examinado: A.R.Q. Paredes 3 (CGMS)

Polygonum meisnerianum Cham. \& Schltdl. Linnaea 3(1): 40-42. 1828 .

Material examinado: Hatschbach, G. 49014 (HUEFS)

Polygonum paraguayense Wedd. Annales des Sciences Naturelles, Botanique, série 3, 13: 253. 1849.

Material examinado: Hatschbach 21979 (MBM)

Polygonum punctatum Elliot. A Sketch of the Botany of South-Carolina and Georgia 1(5): 455-456. 1821[1817]. Material examinado: M. Rocha 206 (CGMS)

Polygonum stelligerum Cham. Linnaea 8: 131. 1833. Material examinado: G.A. Damasceno-Junior 3116 (CGMS, COR)

Ruprechtia C. A. Mey. in Mém. Acad. Imp. Sci. St.Pétersbourg Divers Savans 6: 148-150. 1845 [1840].

De acordo com Pendry (2004), este gênero possui 37 espécies, divididos em árvores, arbustos e lianas. Sua distribuição se dá em toda a região neotropical e subtropical, especialmente na América Latina, tendo como exceção o Chile (Carvalho 2006, Pendry 2003, 2004). O país com maior diversidade de espécies é o Brasil, com 16 espécies aceitas. Para o estado de Mato Grosso do Sul, foram citadas três espécies de Ruprechtia. Uma delas, R. triflora Griseb, no Brasil, ocorre somente no estado, na região do Chaco, mas é citada também para a Bolívia e a Argentina (Pott \& Pott 1994). Com o levantamento, foram encontrados dois novos registros, sendo eles $R$. laxiflora Meisn. e $R$. tenuiflora Benth.

Ruprechtia brachysepala Meisn. Flora Brasiliensis 5(1): 58. 1855. 
Material examinado: Dasmasceno Jr, G. A. 798 (UEC)

Ruprechtia exploratricis Sandwith. Bulletin of Miscellaneous Information Kew 1928: 143. 1928.

Material examinado: D.R.C. Padilha 48 (CGMS)

Ruprechtia laxiflora Meisn. Flora Brasiliensis 5(1): 56. 1855.

Material examinado: G. Hatschbach \& R. Kummrow 48434 (MOBOT BR)

Ruprechtia tenuiflora Benth. London Journal of Botany 4: 629-630. 1845.

Material examinado: J. A. Ratter R5954 (NYBG_BR)

Ruprechtia triflora Griseb. Abhandlungen der Königlichen Gesellschaft der Wissenschaften zu Göttingen 24: 89. 1879. Material examinado: D.R.C. Padilha 65 (CGMS)

Triplaris Loefl. in Iter Hispan. 229, 256. 1758.

As espécies desse gênero distribuem-se de Oaxaca, no México, até o estado do Paraná, no Brasil (Kubitzki et al. 1993). Algumas dessas espécies são conhecidas por pau-de-formiga, pois possuem o caule oco, cujo interior é habitado por algumas espécies de formiga e são, geralmente, utilizadas na ornamentação e na arborização urbana (Junior et al. 2009, Kraus 2005, Lorenzi 2000). No Brasil, de acordo com a LEFB (Melo 2012), encontram-se sete espécies aceitas, e no Mato Grosso do Sul, duas. Elas ocorrem na maior parte das vezes, no Chaco, em Mata Ciliar.

Triplaris americana L. Systema Naturae, Editio Decima 2: 881. 1759.

Material examinado: L.M.M. Arakaki 1 (CGMS)

Triplaris gardneriana Wedd. Annales des Sciences Naturelles; Botanique, sér. 3 13: 265. 1849.

Material examinado: T.L.B.M. Borine 16 (CGMS)

\section{AGRADECIMENTOS}

À todas as pessoas que colaboraram no levantamento de dados, principalmente àquelas do Herbário CGMS . Agradecemos, também, ao Herbário Virtual da Flora e Fungos, pelo trabalho de integração das informações dos acervos dos herbários do país, e ao Conselho Nacional de Desenvolvimento Científico e Tecnológico, pelas bolsas de Iniciação Científica concedidas à primeira e segunda autoras.

\section{REFERÊNCIAS}

Alves, T.M.A., Ribeiro, F.L., Kloos, H. \& Zani, C.L. 2001. Polygodial, the Fungitoxic Component from the Brazilian medicinal plant Polygonum punctatum. Memórias do Instituto Oswaldo Cruz 96(6):831-833.

Angiosperm Phylogeny Group -APG III. 2009. An update of the Angiosperm Phylogeny Group classification for the orders and families of flowering plants: APG III - The Angiosperm Phylogeny Group. Botanical Journal of the Linnean Society 161(2):105-121.
Barros, I.B. de, Daniel, J.F. de S., Pinto, J.P., Rezende, M.I., Filho, R.B. \& Ferreira, D.T. 2011. Phytochemical and antifungal activity of Anthraquinones and root and leaf extracts of Coccoloba molis on phytopathogens. Brazilian Archives of Biology and Technology 54(3):535-541.

Cardoso, C.A.L., Honda, N.K. \& Dias, E.S. 2006. Avaliação do perfil cromatográfico em espécies de Polygonum e amostras comercializadas como "erva-de-bicho". Revista Brasileira de Farmacognosia $16(2): 236-245$

Carvalho, P.E.R. 2006. Marmaleiro-Bravo. Circular Técnica, 122. Embrapa Florestas.

Cornejo, F. \& Janovec, J. 2010. Seeds of Amazonian plants. Princeton Field Guides. Princeton University Press, Princeton-NJ. 192 p.

Dubs, B. 1998. Prodromus Florae Matogrossensis. - Part I. Checklist of Angiosperm, Part II. Types from Mato Grosso. Betrona-Verlag, Küsnacht. 103 p.

Farinaccio, M.A. \& Nascimento, S.M.M. 2005. Plumbaginaceae. In Flora fanerogâmica do estado de São Paulo (M.D.G.L. Wanderley, G.J. Shepherd, T.S.A. Melhem, A.M. Giulietti, S.E. Martins \& M. Kirizawa, org.). Fundação de Amparo à Pesquisa do Estado de São, São Paulo, p 321-322

Forzza, R.C., Filardi, F.L.R., Costa, A., Carvalho, JR, A.A., Peixoto, A.L., Walter, B.M.T., Bicudo, C., Zappi, D., Costa, D.P., Lleras, E., Martinelli, G., Lima, A.C., Prado, J., Stehmann, J.R., Baumgratz, J.F.A., Pirani, J.R., Sylvestre, L.S., Maia, L.C., Lohmann, L.G., Paganucci, L., Silveira, M., Nadruz, M., Mamede, M.C.H., Bastos, M.N.C., Morim, M.P., Barbosa, M.R., Menezes, M., Hopkins, M., Secco, R., Cavalcanti, T. \& Souza, V.C. Lista de Espécies da Flora do Brasil 2012. Disponível em: http://floradobrasil.jbrj.gov.br/2012. Acessado em 25.07.2012.

Jácome, R.L.R.P., Lopes, D.E.S., Recio, R.A., Macedo, J.F. \& Oliveira, A.B. 2004. Caracterização farmacognóstica de Polygonum hydropiperoides Michaux e P. spectabile (Mart.) (Polygonaceae). Revista Brasileira de Farmacognosia 14(1):21-27.

Junior, V.H., Bicudo, L.R.H. \& Fransozo, A. 2009. The Triplaria tree (Triplaris spp.) and Pseudomyrmex ants: a symbiotic with risks of attack for humans. Revista da Sociedade Brasileira de Medicina Tropical 42(6):727-729

Kraus, J.E., Höfling, E., Rodrigues, M.T. \& Sampaio, M.R.A. de. 2005. Fauna e flora no campus da cidade universitária Armando de Salles Oliveira. Instituto de Biociências da Universidade de São Paulo, São Paulo. 311p.

Kubitzki, K., Rohwer, J.G. \& Bittrich, V. 1993. Flowering plants: Dicotyledons, Magnoliid, Hamamelid and Caryophyllid Families. (The Families and genera of vascular plants: 2). Springer-Verlag Berlin Heidelberg, New York. 653 p.

Lorenzi, H. 2000. Árvores Brasileiras: manual de identificação de plantas nativas do Brasil. Editora Plantarum, Nova Odessa. 352 p.

Melo, E. de. 1996. O gênero Polygonum L. (Polygonaceae) no estado da Bahia, Brasil. Sitientibus, 14:45-55

2000. Polygonaceae da Cadeia do Espinhaço, Brasil. Acta Botânica Brasilica 14(3):273-300.

2003. Revisão das espécies do gênero Coccoloba P. Brown (Polygonaceae) para o Brasil. Tese 418 f., Universidade de São Paulo. São Paulo.

2004. As espécies de Coccoloba P. Browne (Polygonaceae) da Amazônia brasileira. Acta Amazonica 34(4):525-551

2012. Polygonaceae In Lista de Espécies da Flora do Brasil. Jardim Botânico do Rio de Janeiro. Disponível em: http://floradobrasil. jbrj.gov.br/2012/FB000196. Acessado em 25.07.2012.

Moore, M.J., Hassan, N., Gitzendanner, M.A., Bruenn, R.A., Croley, M., Vandeventer, A., Horn, J.W, Dhingra, A., Brockington, S.F., Latvis, M., Ramdial, J., Alexandre, R., Piedrahita, A., XI, Z. \& Davis, C.C., Pamela S. Soltis, P.S. \& Soltis, D.E. 2011. Phylogenetic analysis of the plastid inverted repeat for 244 species: insights into deeper-lever angiosperm relationships from a long, slowly evolving sequence region. International Journal of Plant Sciences 172(4):541-558

Oliveira, P.E.S., Santos, W.S. dos, Conserva, L.M. \& Lemos, R.P. de L. 2008. Constituintes químicos das folhas e do caule de Coccoloba mollis Casaretto (Polygonaceae). Revista Brasileira de Farmacognosia 18:713-717 
Olmstead, R.G., Michaels, H.J., Scott, K.M. \& Palmer, J.D. 1992. Monophyly of the Asteridae and identification of their major lineages inferred from DNA sequence of rbcL. Annals of the Missouri Botanical Garden 79:249-265.

Paiva, S.R, Fontoura, L.A. \& Figueiredo, M. R. 2002. Perfil Cromatográfico de duas espécies de Plumbaginaceae: Plumbago scandens L. e Plumbago auriculata. Lam. Química Nova 25(5):717-721

Pendry, C.A. 2003. Nine new species of Ruprechtia (Polygonaceae) from Central and South America. Edinburgh Journal of Botany 60:19-42 . 2004. Monograph of Ruprechtia (Polygonaceae). Systematic Botany Monographs 67:1- 113.

Pott, A., \& Pott, V. J. 1994. Plantas do pantanal. Centro de Pesquisa Agropecuária do Pantanal, Serviço de Produção de Informação. EMBRAPA, Corumbá. 36 p.

Souza, V.C. \& Lorenzi, H. 2008. Botânica sistemática: guia ilustrado para identificação das famílias de fanerógamas nativas e exóticas no Brasil, baseado em APG II. 2. Plantarum, Nova Odessa. 704 p.
Splink. Species Link. Disponível em: <http://www.splink.org.br>. Acessado em 14.08.2012.

Thiers, B. 2008 [continuamente atualizado]. Index Herbariorum: A global directory of public herbaria and associated staff. New York Botanical Garden's Virtual Herbarium. Disponível em: http://sweetgum.nybg. org/ih/. Acessado em 21.07.2012.

Tropicos. 2012. Missouri Botanical Garden. Disponível em: http://www. tropicos.org/Name/26000380. Acessado em: 30.07.2012.

Tsuboy, M.S., Marcarini, J.C., Ferreira, D.T., Ferraz, E.R.A., Chequer, F.M.D., Oliveira, D.P. de, Ribeiro, L.R. \& Mantovani, M.S. 2010. Evaluation of extracts from Coccoloba mollis using the Salmonello/ microsome system and in vivo tests. Genetics and Molecular Biology 33(3):542-548

Zappi, D. 2012. Plumbaginaceae In Lista de Espécies da Flora do Brasil. Jardim Botânico do Rio de Janeiro. Disponível em: $<$ http:// floradobrasil.jbrj.gov.br/2012/FB00019>. Acessado em 25.07.2012. 